\title{
Future Water: Comparing and contrasting approaches to predicting water quality
}

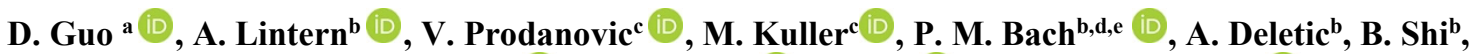

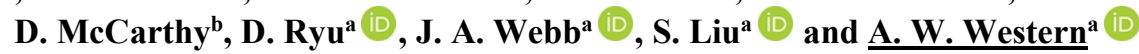 \\ ${ }^{a}$ Department of Infrastructure Engineering, The University of Melbourne, Parkville, VIC Australia \\ ${ }^{b}$ Department of Civil Engineering, 23 College Walk, Monash University, Clayton, VIC Australia \\ ${ }^{c}$ School of Civil and Environmental Engineering, Faculty of Engineering, UNSW Sydney, NSW Australia \\ ${ }^{d}$ Swiss Federal Institute of Aquatic Science \& Technology (Eawag), Dübendorf 8600, Switzerland \\ ${ }^{e}$ Institute of Environmental Engineering, ETH Zürich, 8093, Zürich, Switzerland
}

Email:danlu.guo@unimelb.edu.au

\begin{abstract}
Globally, surface water quality deterioration is an important issue exacerbated by increasing urbanisation, intensified agricultural activities and climate change. To mitigate this increase in waterway pollution there is a strong need for effective catchment management strategies. However, this is currently limited by: (1) our lack of understanding of the key processes and mechanisms driving water quality change in waterways, and (2) our inability to predict future water quality change. To address these, we need to improve our ability to understand and model water quality. This improved capacity will enable us to better identify important water quality changes under various land use, land management and climate scenarios. These improvements could thus assist waterway managers to better prioritise regions which require special management attention, as well as better assess the benefits of different management interventions and preventative actions to reduce pollution levels.
\end{abstract}

Given the above, there is a vital role for predictive models for supporting catchment water quality management. There is a wide spectrum of surface water quality modelling approaches, ranging from purely black-box empirical to detailed process-oriented models; these often have contrasting strengths and weaknesses. This study aims to (1) provide a review of key considerations for designing and developing effective modelling approaches for water quality prediction; and to (2) outline reasons for choices in modelling approaches for rural and urban water quality across Victoria. A fundamental consideration is the modelling purpose which provides preliminary guidelines for other modelling decisions. The core decision in modelling water quality is the conceptualisation of key physical processes, which involves the definition of pollution sources, flow/transport pathways and other catchment features. In addition, practical considerations such as data availability and model development effort are also important. For each consideration we provide recommendations for different types of catchments, and specifically highlight distinctions between natural/rural and urban catchments.

We illustrate these considerations with two modelling approaches: a) a data-driven statistical approach informed by conceptual understanding and b) a more process-oriented approach. These models have been applied in rural and urban settings respectively. Both approaches are designed to identify spatio-temporal differences in water quality, and to predict how future water quality will change. Being designed for vastly different environments (rural vs urban), these two models present separate, yet parallel approaches for modelling spatio-temporal variability in water quality. By contrasting these models, we aim to highlight strengths and weaknesses of different approaches, to share practical experiences from implementing these approaches and to identify the ways forward for both approaches. The experiences and recommendations from these modelling approaches can provide valuable recommendations to assist future water quality modelling works to identify effective modelling approaches.

Keywords: $\quad$ Water quality modelling, urban catchments, rural catchments, sediments, nutrients 
Guo et al., Predicting Future Water: Comparing and contrasting approaches to predicting future water quality

\section{INTRODUCTION}

Waterways throughout the world are experiencing deteriorating water quality with increasing levels of urbanisation, climate change and agricultural intensification (Schwarzenbach et al. 2010). Previous studies have shown that most waterways in Australia are receiving greater nutrient and sediment loads compared to preEuropean states (National Water Commission 2005). Due to increasing water scarcity in the future as well as to protect environmental values, there is a need to mitigate this increase in waterway pollution.

Among other barriers, successful management of waterway pollution is hindered by two major knowledge gaps: (1) our lack of understanding of the key processes and mechanisms driving water quality change in waterways, and (2) our inability to predict future water quality change. Being able to predict future water quality changes under various climate change, land use change, and land and water management strategy implementation scenarios will enable waterway managers to better prioritise regions for catchment management, and to identify the best management practices required to reduce pollution levels. Predictive models of water quality are therefore vital for successful catchment management.

There is a wide spectrum of surface water quality modelling approaches from purely black-box empirical to detailed process-oriented models, which often have contrasting strengths and weaknesses regarding e.g., capacities to represent key physical processes, requirements for data and model development effort. In this paper we discuss some key aspects that need to be considered when designing and developing effective modelling approaches for water quality prediction. We then illustrate these considerations adoption of two modelling approaches. These include: a) a data-driven statistical approach informed by conceptual understanding and b) a more process-oriented approach. These two approaches have been applied in rural and urban settings respectively and are being developed with a view to improving Victoria's future water modelling capability.

\section{KEY CONSIDERATIONS FOR MODEL CHOICE}

Effective modelling of surface water quality requires consideration of multiple factors when conceptualising, developing and implementing the model, which will ultimately influence model performance and interpretability. As a general principle of modelling, the intended purpose of a model is fundamental to all other model considerations. Specific to surface water quality, modelling capacity is dependent on good conceptualisation of the key physical processes involved. This requires identifying the important pollution sources, flow pathways and other catchment features and decisions on how they are represented in the model. Furthermore, spatial and temporal resolutions at which these processes should be captured are also critical. Last but not least, regarding practical implementation, all the above-mentioned desirables need to be supported by matching levels of data availability and model development and testing effort.

\subsection{Modelling purpose}

Surface water quality models are developed for different purposes, such as to estimate pollutant load delivered to a receiving water body, to understand the impact of agricultural/industrial sectors on water quality, to capture water quality changes under altered hydro-climatic conditions, or to explore how water quality is affected by urban development. In addition, in contrast to 'traditional' modelling methods, novel models are moving towards 'supporting' decision making process, rather than simply 'predicting' an outcome. The intended purpose of a water quality model will impact on choices made about its design, as well as the numerical simplifications required to make the model robust and fast. Hence, surface water quality modelling usually adopts a "fit-for-purpose" approach, which answers a specific need, rather than trying to model all processes across multiple catchments.

Often the model's purpose will also define the spatial extent of modelling; e.g. to understand water quality trends in an environmentally significant region. Microscale models are able to reliably predict pollution build-up and wash-off from a single surface (e.g. roof surfaces, Egodawatta et al., 2009); however, they are often not usable in a precinct or at the catchment scale, due to their complexity. On the other hand, mesoscale or catchment-scale models use generalised forms of build-up and wash-off equations, calibrating pollution models only on certain points in the catchment or linking pollution to specific land uses (e.g. modelling pollution in commonly used EPA SWMM model, Tsihrintzis and Hamid (1998)).

The level of detail or processes that are captured in a model can also be dependent on whether the model is used to interrogate past water quality events or to predict future quality. While both can be designed to account for all pollution-driven processes, future prediction models often simplify processes to achieve the necessary robustness and lower uncertainty (Beck, 1987). Simple models are also more easily integrated with other sub-models (e.g. for water supply, stormwater, water treatment) (Bach et al., 2014). Similarly, selection of input water quality monitoring data sets (event-based or continuous data) for the model are highly dependent on the complexity and 
spatial scale of the physical processes being modelled. For constituents that required complex or expensive analytical methods (such as stormwater reference pathogens) or have high spatial variability, event-based monitoring data sets are often utilised due to limited availability of continuous data (McCarthy et al., 2011). With increasing data collection in urban and peri-urban areas, there is a shift towards continuous modelling approaches; however, this requires increased complexity of the simulation, which might not be suitable for some purposes. The overall modelling purpose sets the context, which often provides preliminary guidelines and sets 'boundary conditions' to other specific considerations on model development (discussed below).

\subsection{Conceptualisation of key physical processes}

In water quality modelling, the source, mobilisation and delivery of pollutants can be represented in different ways, which may be better suited to different situations. Regarding the conceptualisation of these processes, the relevant modelling considerations can be categorised into the following four groups, which are: (1) pollution sources, (2) flow pathways, (3) other catchment features and (4) spatial and temporal resolution. Each consideration is listed will be discussed in detail subsequently.

\section{Pollution sources}

Pollutants and constituents such as nutrients, sediments, salts and metals can come from a variety of sources in both rural and urban catchments, depending on the specific land use (Table 1). Whilst Table 1 provides a summary of the typical and diffuse point sources of pollutants in urban and rural catchments, the importance of these sources depends significantly on factors such as: population density, industrial land use (and industry type), traffic volumes and construction activities (in urban catchments) (Shi et al. 2019); and crop type, stocking densities and land management strategies (in rural areas) (Lintern et al. 2018a). These contrasting and specific pollution sources need to be captured in water quality models of rural and urban catchments.

Table 1. Point and diffuse pollution sources in urban and rural areas

\begin{tabular}{|l|l|l|}
\hline & Point Sources & Diffuse Sources \\
\hline Urban & Industrial discharge & Atmospheric Deposition \\
& Wastewater treatment plant discharge & Stormwater runoff \\
& Combined sewer overflows (CSO) & Construction activities \\
& & Fertiliser application \\
& & Road Deposition \\
& & Leaching of street furniture \\
\hline Rural & Effluent pond discharge & Animal/household pet manure \\
& Tile drains & Atmospheric Deposition \\
& Irrigation return flow & Fertiliser application \\
& Track and road runoff & Sediment erosion \\
& Mining activities (historic and current) & Livestock manure \\
& Pesticide application \\
\hline
\end{tabular}

Furthermore, constituents of concern requiring monitoring and modelling may differ between catchments due to different pollution sources within these catchments. Whilst metals (e.g., copper, lead and zinc) are more likely to be of concern in urban catchments with high levels of industry, pesticides (e.g., glyphosate) may be of concern in urban catchments with high levels of low-density residential dwellings and in rural cropping catchments. Similarly, whilst nitrogen may be of concern in intensively cropped rural catchments, sediments may be the focus of key modelling efforts in rural areas used for cattle grazing.

\section{Flow pathways}

Constituent transport and transformation is strongly dependent on hydrologic (soil water) conditions and hydrologic flow pathways. These depend on both catchment characteristics (Kirkby, 1988) and variations in the wetness condition of the catchment over time (Western, et al., 1999), often leading to thresholds in runoff response (Detty and McGuire, 2010, Janzen and McDonnell, 2015, Saffarpour, et al., 2016). A good example for such complexity is the so-called double paradox (typically in small catchments), where streamflow responds rapidly to rainfall events, but waterway chemicals are strongly dominated by old water (Bishop, et al., 2004; Kirchner, 2003). Clear understanding of flow pathways and pollutant sources within the catchment are keys to represent these phenomena in water quality modelling. Urban environments have the added complication of flow pathway alterations resulting from development of impervious surfaces, artificial drainage conduits and development of new preferential flow pathways associated with urban infrastructure (Bonneau, et al., 2017).

This has various implications for different modelling approaches. In statistical approaches, it suggests including predictors such as terrain, climate and soil characteristics that are predictors of runoff processes and pathways. For process-oriented approaches, better water quality predictions require a sound understanding of transport pathways 
Guo et al., Predicting Future Water: Comparing and contrasting approaches to predicting future water quality

and transit times; however, an important issue is that traditional rainfall-runoff models often poorly represent both flow paths and transit times (Hrachowitz, et al., 2016). Progress is being made in coupling flow and (solute transport) transit time responses (Benettin and Bertuzzo, 2018, Rinaldo, et al., 2015).

\section{Other catchment features}

There are other catchment features that may need to be included in water quality models. Climate, topography, vegetation cover, geology, soil conditions and artificial drainage infrastructure can all affect pollutant runoff processes, sources and transformations (e.g., denitrification, nutrient mineralisation from organic matter) in both urban and rural catchments (Shi et al. 2019, Lintern et al. 2018a). While the importance of these various catchment characteristics on pollutant levels in receiving water has been assessed in rural and urban catchments, a comprehensive comparison of the relative importance of these landscape features has not been conducted.

Water quality management strategies (e.g., Best Management Practices, Low Impact Development) have been extensively implemented in both rural and urban catchments. These strategies can be either physically built, or focused on changing social behaviour (Lintern et al. 2018a). However, the specific behaviours targeted, and the specific structures built vary between urban and rural environments. For sediment load reduction, for example, street sweeping may be utilised in urban catchments (Zhao et al. 2010), but reduction in stocking density or fencing to restrict stock access to streams may be utilised in rural catchments (McKergrow et al. 2003).

\section{Spatial and temporal resolution of interest}

The spatial and temporal resolution of a model should be determined by the modelling purpose, while also reflecting the key processes and catchment characteristics. Generally speaking, spatial diversity of diffuse pollution sources and catchment characteristics such as land-use is higher for urban catchments compared to rural areas. Furthermore, point sources such as combined sewer overflows and industry discharges play a significant role in urban catchments, directly impacting surface water quality in a specific location. Depending on the purpose, spatial resolution of urban pollution modelling can go from as fine as individual buildings or plots up to the urban stream/river basin (from tens of several square metres to several square kilometres). For rural catchments, which have low imperviousness, individual plots are of less interest; pollution sources tend to be diffuse and homogenous over larger areas. Here, the spatial scale may vary from small creek catchments up to large river basins spanning multiple states, often with hydrologic response units used to capture landuse and other variations within subcatchments.

A model's temporal resolution should reflect the rate of physical (or sometimes chemical) processes occurring in the study area, which often differ between urban and rural catchments and also change over time. For urban catchments with high imperviousness, diffuse pollution from stormwater runoff can affect surface water quality within minutes of the start of a rainfall event (often 1 to 6 minutes, depending on the rainfall intensity, Fletcher et al. (2013)). Depending on the characteristics of the urban drainage and wastewater systems, diurnal patterns of domestic and industrial water use affect surface water quality on a sub-daily level, while point sources may immediately pollute urban waterways. During dry periods, the time-step increases to an hour or more, when there is no significant stormwater pollution transport through the catchment (assuming minimal dry weather flows in stormwater drainage network). For rural areas, diffuse pollution from rainfall and runoff, although retarded and infiltrated before reaching surface waters, can result in sub-daily fluctuations of pollution loadings, often at similar timescales to runoff events. Monthly and seasonal scales play a bigger role in rural than urban catchments, due to hydrologic seasonality, plant growth and agricultural cycles. To explore the impacts of climate change, land use change (e.g. urbanisation, agricultural intensification) and best management practice implementation, very long timescales of decades are relevant to both urban and rural pollution models.

\subsection{Data availability and model development effort}

Generally speaking, water quality data availability can be patchy over space and time, as well as for different constituents, due to the local/regional monitoring programs and a wide range of management interests. Large-scale datasets on some of the more common constituents (e.g. salts, sediments, nutrients, dissolved oxygen (DO)) are often managed by state government agencies. In contrast, for constituents with of narrower concern, available data tend to cluster in small areas that are of concern for local utilities and catchment managers (e.g. heavy metals from mining districts). It is common for data to be collected at regular (e.g. monthly) intervals, although event-based monitoring is also implemented, such as for the Paddock to Reef Integrated Monitoring, Modelling and Reporting Program in the Great Barrier Reef catchments (Turner et al., 2012).

Model input data (pollution sources, catchment features and flow pathways) are often poorly defined with coarser resolution in natural/rural catchments, and even further limited in ungauged catchments. Their information content may be insufficient to drive sophisticated process-based models especially over large regions; alternatively, 
statistical models may make better use of the limited data while also reducing effort for model development and calibration. In contrast, urban catchments generally have more well-defined sources, features and flow pathways including discharge points, impervious areas and other infrastructure (e.g. sewers). The relatively more complex catchment characteristics also require potentially more effort for data compilation and validation; consequently, models are often developed in a local context.

\section{TWO CONTRASTING MODELLING APPROACHES}

We introduce two water quality modelling approaches that we have used in previous studies. The key purposes of these two models differ as they are focussed on a) understanding key causes of water quality changes and to make predictions for rural catchments; and b) predicting future changes of water quality in urban catchments.

\subsection{Understanding and predicting water quality in rural catchments}

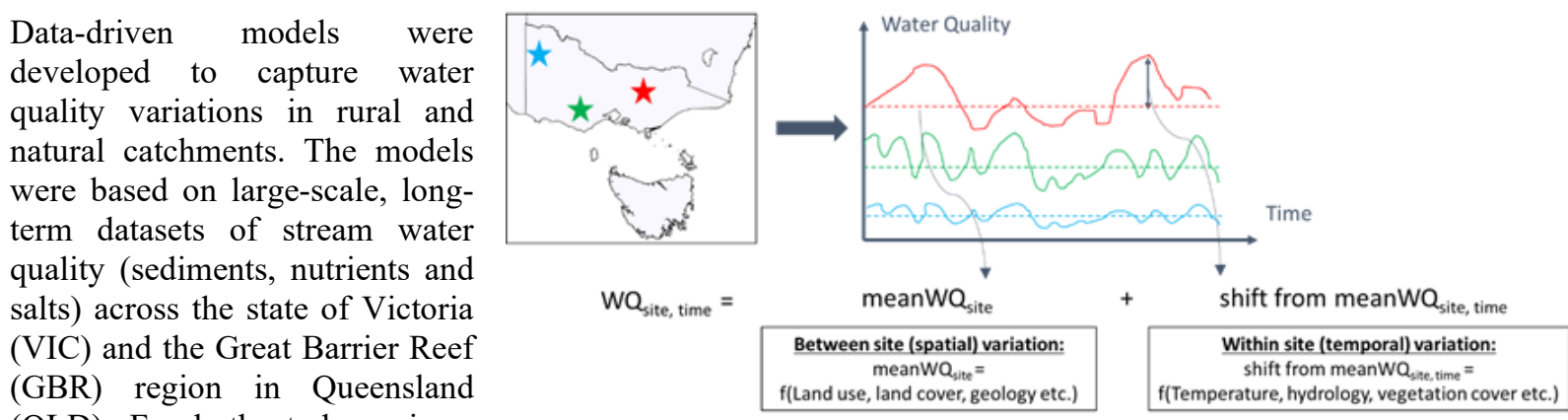
(QLD). For both study regions, our models focused on how water

Figure 1. Schematic of the rural water quality model quality (i.e. the concentration of each constituent considered) varies: a) over space; and b) over time (across individual spot samples for VIC, and across different events for QLD using event-mean concentrations). We developed two corresponding models within the overall hierarchical framework: a) a model that relates the spatial variability of catchment-level mean concentrations to variations in catchment characteristics over space; and b) a model that relates the temporal shift of concentrations from the catchment level means to changes in the climatic, hydrologic and vegetation conditions over time. The models represent the key controls that affect water quality in rural catchments with predictors that vary both spatially and temporally, with the magnitude of these impacts determined by long-term datasets.

The model predictors were determined using a data-driven approach that is informed by process conceptualisations. This started with a comprehensive literature review to identify the key controls and processes that influence the source, mobilisation and transport of river pollutants (Lintern et al., 2018a), and thus inform the potential predictors for our models. We evaluated all combinations of a large number of possible predictors to identify the optimal sets of model predictors with the highest relative predictive powers.

For each constituent modelled, the key predictors that describe the spatial and temporal variabilities were selected separately. When representing spatial variability in stream water quality, we identified important key predictors of: natural catchment characteristics (topography, climate, soil type), as well as agricultural activities - particularly cropping and pasture in VIC (Lintern et al., 2018b), and sugarcane in the GBR catchments. Temporal variability was largely driven by changes in same-day and antecedent streamflow, as well as soil moisture and vegetation cover (Guo et al., 2019). Both models employed a linear additive model structure with data transformation. The impacts of the selected key predictors differed by constituent, indicating potentially different mobilisation/transportation pathways via surface flow, shallow subsurface flow and groundwater flow.

The models captured reasonable proportions of spatial variabilities (over 50\%) in stream water quality, while temporal variabilities were generally less well described (less than 50\%). For both spatial and temporal variability, it was generally more challenging to represent non-conservative constituents. This may have been due to the more complex processes involved beyond those that the model could represent, such as denitrification, plant uptake and soil adsorption. The temporal model performance was also limited for constituents that are often present at low (near detection limit) concentrations. Such data are more likely subject to greater measurement errors after data transformation, which could limit the model capacity to accurately capture variabilities in lower concentrations. 


\subsection{Predicting future water quality in urban catchments}

Urban catchments present highly altered hydrological cycles due to increased imperviousness and human activity. Wash-off of pollution from impervious areas, point source pollution from human activity and sewage leaks are leading to deteriorating water quality. To date, build-up/wash-off models have had limited success in accurately representing pollution

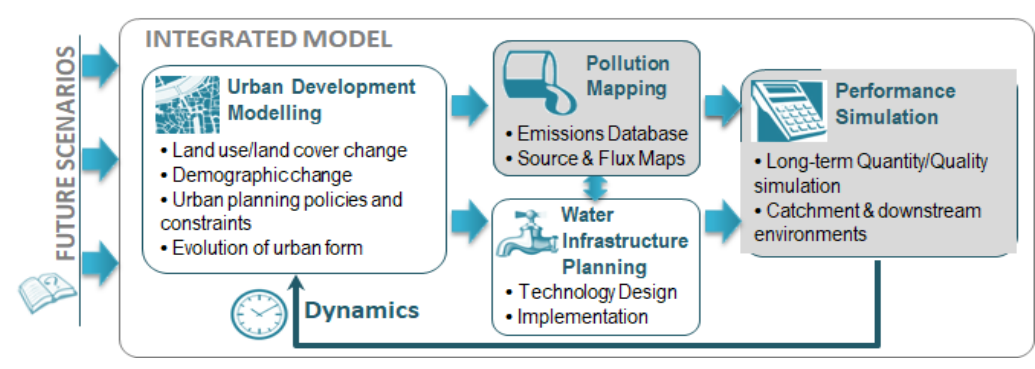

Figure 2. Schematic of the urban water quality model concentrations, and also mostly disregard point-source contribution and spatial distribution of pollution.

A spatially distributed physical model was adopted, which described pollution generation and transport. The model combined a spatial dynamic urban water planning simulation model (UrbanBEATS) to the hydraulic and water quality modules of the widely used stormwater management model (SWMM). The model calculated distributed pollution generation from wash-off based on local land-use mixes, and combined this with unconventional point and distributed sources (Shi et al., 2019). Variability of unconventional pollution sources was also considered.

Key data used include land use (current and/or planned), population density, digital elevation model (DEM), information on potential point-sources, rain series and potential infiltration and evaporation. Calibration was done on both hydraulic and pollution models using land use parameters, and pollution build-up and wash-off coefficients.

The dynamic functionality of UrbanBEATS allowed us to run scenario-based predictions for existing urban areas. Moreover, our model enableed exploration of the effect of urban expansion and pollution from future development, as well as the effectiveness of mitigation strategies such as Water Sensitive Urban Design (WSUD).

Further work includes the calibration of the pollution model, as well as the development of the model for unconventional point-source pollution generation. Once this is accomplished, the model will be tested on historical data from a real case. Finally, improvements in simulation speed are necessary to improve the model's fitness for rapid scenario analysis.

\section{CONCLUDING HIGHLIGHTS AND WAYS FORWARD}

The rural and urban models have their own strengths and weaknesses. The rural model is based on relationships within historical data; although we used a conceptual understanding of water quality processes to underpin the datasets, our model is still limited by our assumption that historical processes will hold in the future. However, this is likely not the case considering possible future changes in land use, land management and climate. One potential way to better understand the model's prediction capacity is via extensive calibration and validation to cover a wide range of land use and climatic conditions. This approach would quantify uncertainties and define 'safe boundaries' when using the model to make predictions. The urban model is more process-based, but the forecasts produced by the model are still difficult to validate because there are future scenarios that have not yet occurred. In contrast, hindcasts can usually be calibrated easily if the requisite complex datasets are available. Cross validation of these forecasts using different methods and models would increase the robustness of these forecasts. To better facilitate scenario testing, the urban model should also be improved in terms of rapid simulation. This can be potentially achieved by simplifying urban process representation and thus the gross data requirements.

In prediction mode, the urban model would be better suited to explore water quality responses to changes related to infrastructure, development and population growth, whereas the rural model would be more useful to explore changes associated with the conditions of catchments (e.g. climate and anthropogenic changes in land use). These models can be used together to gain a whole-of-catchment understanding of water quality responses. For example, to model the Greater Melbourne region, we need not only the urban model to provide good representation of water quality in the metropolitan region, but also the rural model to understand riverine water quality upstream of the metropolitan region, and thus to identify an upstream boundary condition for the urban model.

\section{ACKNOWLEDGMENTS}

The rural and urban models introduced were funded by the Australian Research Council (LP140100495 and LP160100241) and Environment Protection Authority (VIC), Melbourne Water, Knox City Council, Department 
Guo et al., Predicting Future Water: Comparing and contrasting approaches to predicting future water quality

of Environment, Land, Water and Planning (VIC), Bureau of Meteorology and Department of Natural Resources, Mines and Energy (QLD).

\section{REFERENCES}

Bach, P. M., Rauch, W., Mikkelsen, P. S., McCarthy, D. T., and Deletic, A. (2014). A critical review of integrated urban water modelling - Urban drainage and beyond, Environmental Modelling \& Software, 54, 88-107, https://doi.org/10.1016/j.envsoft.2013.12.018.

Beck, M. B. (1987). Water quality modeling: A review of the analysis of uncertainty. Water Resources Research, 23, $1393-$ 1442.

Benettin, P., Bertuzzo, E. (2018) tran-SAS v1.0: a numerical model to compute catchment-scale hydrologic transport using StorAge Selection functions. Geoscientific Model Development 11: 1627-1639 DOI 10.5194/gmd-11-1627-2018

Bishop, K., Seibert, J., Köhler, S., Laudon, H. (2004) Resolving the Double Paradox of rapidly mobilized old water with highly variable responses in runoff chemistry. Hydrological Processes 18: 185-189 DOI 10.1002/hyp.5209

Bonneau, J., Fletcher, T. D., Costelloe J. F., Burns, M. J. (2017) Stormwater infiltration and the 'urban karst' - A review. Journal of Hydrology 552: 141-150 DOI 10.1016/j.jhydrol.2017.06.043

Detty, J. M., McGuire, K. J. (2010) Threshold changes in storm runoff generation at a till-mantled headwater catchment. Water Resources Research 46: W07525, doi 07510.01029/02009WR008102

Egodawatta, P., Thomas, E. and Goonetilleke, A. (2009). Understanding the physical processes of pollutant build-up and washoff on roof surfaces. Science of The Total Environment, 407, 1834-41.

Fletcher, T. D., Andrieu, H. and Hamel, P. (2013). Understanding, management and modelling of urban hydrology and its consequences for receiving waters: A state of the art. Advances in Water Resources, 51, 261-279.

Guo, D., Lintern, A., Webb, J. A., Ryu, D., Liu, S., Bende-Michl, U., Leahy, P., Wilson, P. and Western, A. W. (2019). Key Factors Affecting Temporal Variability in Stream Water Quality. Water Resources Research, 55, 112-129.

Hrachowitz, M., Benettin, P., van Breukelen, B. M., Fovet, O., Howden, N. J. K., Ruiz, L., van der Velde, Y., Wade, A. J. (2016) Transit times-the link between hydrology and water quality at the catchment scale. Wiley Interdisciplinary Reviews: Water 3: 629-657 DOI 10.1002/wat2.1155

Janzen, D., McDonnell, J. J. (2015) A stochastic approach to modelling and understanding hillslope runoff connectivity dynamics. Ecol Modell 298: 64-74 DOI http://dx.doi.org/10.1016/j.ecolmodel.2014.06.024

Kirchner, J. W. (2003) A double paradox in catchment hydrology and geochemistry. Hydrological Processes 17: 871-874 DOI 10.1002/hyp.5108

Kirkby, M. (1988) Hillslope runoff processes and models. Journal of Hydrology 100: 315-339

Lintern, A., Webb, J. A., Ryu, D., Liu, S., Bende-Michl, U., Waters, D., Leahy, P., Wilson, P. and Western, A. W. (2018a). Key Factors Influencing Differences in Stream Water Quality across Space. Wires Water 51 (1): e1260.

Lintern, A., Webb, J. A., Ryu, D., Liu, S., Waters, D., Leahy, P., Bende-Michl, U., and Western, A. W. (2018b): What Are the Key Catchment Characteristics Affecting Spatial Differences in Riverine Water Quality?, Water Resources Research, doi:10.1029/2017WR022172.

McCarthy, D. T., Deletic, A., Mitchell, V. G. and Diaper, C. (2011). Development and testing of a model for Micro-Organism Prediction in Urban Stormwater (MOPUS). Journal of Hydrology, 409, 236-247.

McKergow, L. A, Weaver, D. M., Prosser, I. P., Grayson, R. B. and Reed A. E. G. (2003. Before and after Riparian Management: Sediment and Nutrient Exports from a Small Agricultural Catchment, Western Australia. Journal of Hydrology 270 (3-4): 253-72. https://doi.org/http://dx.doi.org/10.1016/S0022-1694(02)00286-X.

National Water Commission. 2005. Australian Water Resources 2005. Canberra.

Rinaldo, A., Benettin, P., Harman, C. J., Hrachowitz, M., McGuire, K. J., van der Velde, Y., Bertuzzo, E., Botter, G. (2015) Storage selection functions: A coherent framework for quantifying how catchments store and release water and solutes. Water Resources Research 51: 4840-4847 DOI 10.1002/2015wr017273

Saffarpour, S., Western, A. W., Adams, R., McDonnell, J. J. (2016) Multiple runoff processes and multiple thresholds control agricultural runoff generation. Hydrology and Earth System Science 20: 4525-4545 DOI 10.5194/hess-20-4525-2016

Schwarzenbach, René P, Thomas Egli, Thomas B Hofstetter, Urs von Gunten, and Bernhard Wehrli. 2010. Global Water Pollution and Human Health. Annual Review of Environment and Resources 35 (1): 109-36. https://doi.org/10.1146/annurev-environ-100809-125342.

Shi, B., Bach, P. M., Lintern, A., Zhang, K., Coleman, R. A., Metzeling, L., McCarthy, D. T. and Deletic, A. (2019). Understanding Spatiotemporal Variability of In-Stream Water Quality in Urban Environments - A Case Study of Melbourne, Australia. Journal of Environmental Management 246: 203-13. https://doi.org/https://doi.org/10.1016/j.jenvman.2019.06.006.

Tsihrintzis, V. A. and Hamid, R. (1998). Runoff quality prediction from small urban catchments using SWMM. Hydrological Processes, 12, 311-329.

Turner, R., Huggins, R., Wallace, R., Smith, R., Vardy, S., Warne, M.S.J., (2012). Sediment, Nutrient and Pesticide Loads: Great Barrier Reef Catchment Loads Monitoring 2009-2010. Department of Science. Information Technology, Innovation and the Arts, Brisbane, pp. 53.

Western, A. W., Grayson, R. B., Blöschl, G., Willgoose, G. R., McMahon, T. A. (1999) Observed spatial organisation of soil moisture and its relation to terrain indices. Water Resources Research 35: 797-810

Zhao, H., Li, X., Wang, X., and Tian, D.. 2010. Grain Size Distribution of Road-Deposited Sediment and Its Contribution to Heavy Metal Pollution in Urban Runoff in Beijing, China. Journal of Hazardous Materials 183 (1-3): 203-10. https://doi.org/https://doi.org/10.1016/j.jhazmat.2010.07.012. 